\title{
Spectroscopy Using Sum- and Difference-Frequency Generation in Molecular Solids
}

\author{
Bernhard Dick and R. M.Hochstrasser \\ Department of Chemistry, University of Pennsylvania, Philadelphia, Pennsylvania 19104
}

(Received 17 October 1983)

\begin{abstract}
Both sum- and difference-frequency generation in organic materials are demonstrated and explored under conditions where all three waves $\left(\omega_{1}\right.$ and $\omega_{2}$ ingoing and $\omega_{1} \pm \omega_{2}$ outgoing) are resonant with molecular transitions. The experiments are carried out in centrosymmetric media in which a small electric field is used to distinguish the polar sublattices. Unique electric field dependences of the parametric signals were discovered. The spectroscopic line narrowing and dephasing-induced coherent-emission resonances of these $\chi^{(2)}$ processes make them useful new spectroscopic methods.
\end{abstract}

PACS numbers: $78.40 . \mathrm{Ha}, 33.55 .+\mathrm{c}, 42.65 . \mathrm{Cq}$

We report the first experimental observation of both sum- and difference-frequency optical parametric processes under fully resonant conditions. These are also the first sum- and difference-generation measurements in molecular solids. The fully resonant condition refers to the outgoing wave and both of the ingoing waves being in resonance with specific vibrational-electronic transitions of the molecular system. These results are the realization of some recent predictions. ${ }^{1}$

Most organic solids that are composed of dipolar molecules are nevertheless centrosymmetric so that the second-order dipole susceptibility $\chi^{(2)}$ vanishes identically. The sublattices formed by translations of each of the polar molecules in the unit cell have a finite $\chi^{(2)}$. The sublattice $\chi^{(2)}$ can be fully utilized in second-order nonlinear processes which involve electromagnetic radiation chosen to be resonant with vibrationalelectronic transitions of only that sublattice. The transitions of the different sublattices are distinguishable in the presence of a dc electric field directed along appropriate crystallographic directions. ${ }^{2}$ The same considerations apply if the polar molecules are substitutionally dissolved in a host lattice consisting of nonpolar molecules. This is the type of system used for the experiments reported here which consist of sum- and difference-frequency generation in the system azulene (the "resonant" polar molecule) in naphthalene (the host) induced by a dc electric field. The relevant level scheme appropriate to the reported experiments consists of the electronic ground state $\left(S_{0}\right)$ of azulene, and the vibrationless levels of the first and second excited electronic states $\left(S_{1}\right.$ and $\left.S_{2}\right)$ of azulene at $14651 \mathrm{~cm}^{-1}$ and $28048 \mathrm{~cm}^{-1}$, respectively. The sum- and difference-frequency experiments correspond to generating light at frequencies near to the $S_{2}-S_{0}$ and $S_{2}-S_{1}$ separations, respectively.

The formulas for the susceptibilities given in Ref. 1 for three-level systems are readily adapted to the present situation by adding the energy shift $\Delta \vec{\mu} \cdot \overrightarrow{\mathrm{E}}$ for each transition and summing over the orientations of the two sublattices. The values of $\Delta \vec{\mu} \cdot \vec{E}$ are known from previous studies of the Stark effect in this mixed crystal. ${ }^{3}$ The susceptibilities for sum- and difference-frequency generation are

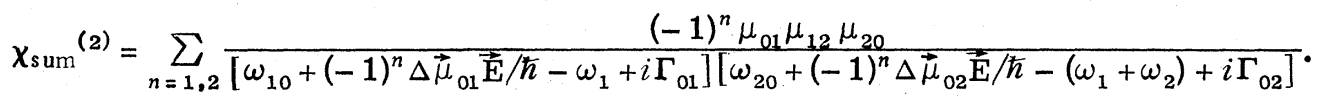

$$
\begin{aligned}
& \chi_{\text {diff }}^{(2)}=\sum_{n=1,2} \frac{(-1)^{n} \mu_{20} \mu_{01} \mu_{12}}{\left[\omega_{10}+(-1)^{n} \Delta \vec{\mu}_{01} \vec{E} / \hbar-\omega_{1}+i \Gamma_{01}\right]\left[\omega_{20}+(-1)^{n} \Delta \vec{\mu}{ }_{02} \vec{E} / \hbar-\omega_{2}-i \Gamma_{02}\right]}
\end{aligned}
$$

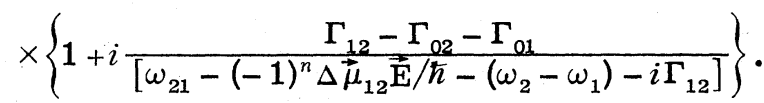

The transition dipoles are denoted by $\mu_{i j}$; the difference in permanent dipole moment is $\Delta \vec{\mu}_{i j}=\vec{\mu}_{i}$ $-\vec{\mu}_{j} ; \omega_{i j}=\omega_{i}-\omega_{j} ;$ and $\Gamma_{i j}$ corresponds to the homogeneous linewidth of the indicated transition. The generated waves are obtained from the square of the ensemble average of $\chi_{\text {sum }}{ }^{(2)}+\chi_{\mathrm{nr}}$ or $\chi_{\text {diff }}{ }^{(2)}$ $+\chi_{\mathrm{nr}}{ }^{(2)}$ where $\chi_{\mathrm{nr}}{ }^{(2)}$ is the nonresonant contribution.

The experimental arrangement consisted of two dye lasers pumped by the same frequency-doubled $\mathrm{Nd}$-doped yttrium aluminum garnet lasers. Both lasers could be narrowed with etalons and pressure 
tuned. Both beams were brought to a common focus on the $a b$ cleavage plane of the naphthalene sample crystal immersed in liquid helium. The static electric field was applied either in the direction of the $b$ axis or, with use of transparent electrodes, perpendicular to the $a b$ face. The latter arrangement gave best results, allowing phase matching by rotation of the crystal around the $b$ axis. Spectra could be obtained with use of other faces and with various polarizations of the laser beams.

With both lasers fixed on resonance, the expression for sum-frequency generation predicts that the signal will first increase quadratically with the applied field just as in conventional electric-field-induced second-harmonic generation. ${ }^{4}$ Figure 1 shows a series of spectra taken with $\omega_{2}$ fixed and $\omega_{1}$ pressure tuned over the $S_{0} \rightarrow S_{1}$ resonance for various settings of the applied field. The inset shows the quadratic behavior of the peak sum-frequency signal in the region of low dc field strength. The higher fields separate the two sublattices completely and the peak intensity becomes independent of the dc field strength, while the signal at the center drops to zero (Fig。1). With both lasers detuned from resonance a nonresonant background signal could be observed which was more than a factor of 1000 weaker than the resonant signal and displayed quadratic dependence on the applied dc field strength. The linewidths of the resonances in Fig. 1 are $0.5 \mathrm{~cm}^{-1}$ corresponding to the inhomogeneous linewidth of the $2 \rightarrow 0$ transition, ${ }^{5}$ the

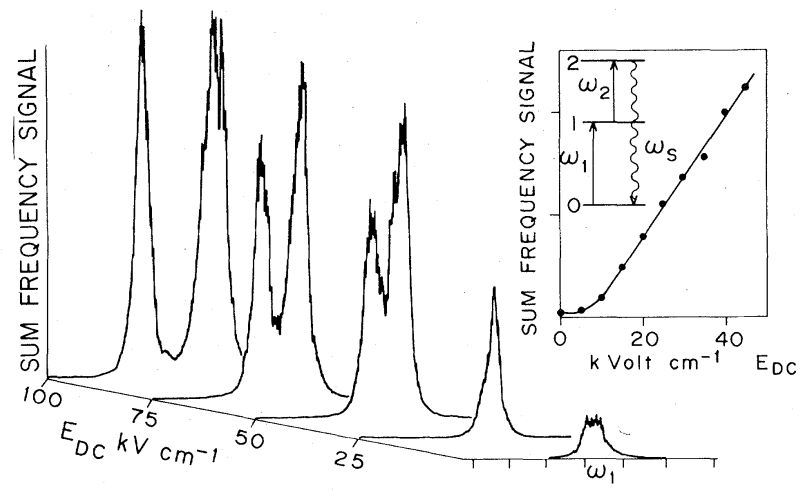

FIG. 1. Resonant sum-frequency generation in dc fields. The $\omega_{1}$ laser scan is shown for various dc fields with $\omega_{2}$ fixed on resonance. (Etalon markers spaced by $1.1 \mathrm{~cm}^{-1}$ are shown on the frequency axis.) Note that the widths of the resonances correspond to the inhomogeneous width of the $S_{0} \rightarrow S_{2}$ transition and not to the width of the $S_{0} \rightarrow S_{1}$ transition over which $\omega_{1}$ is being tuned. Inset: The peak intensity of a sum-frequency signal as a function of dc field strength for low fields. outgoing field resonance.

Spectra similar to those in Fig. 1 were obtained in sum and difference frequency with $\omega_{2}$ scanned. An example is given in Fig. 2 for difference-frequency generation with the dc field chosen to introduce a Stark splitting that is much less than the linewidth. $\omega_{2}$ was scanned for various detunings of $\omega_{1}$. The plot of the peak intensities versus the detuning (inset) reveals a resonance with $3.5 \mathrm{~cm}^{-1}$ width resulting from the twofold convolution of the $2.0-\mathrm{cm}^{-1}$ homogeneous linewidth of the $0 \rightarrow 1$ transition ${ }^{5}$ with the bandwidths of the dye lasers ( $0.7 \mathrm{~cm}^{-1}$ without the etalons). We have also directly observed the $0 \rightarrow 1$ resonance in both sum- and difference-frequency generation using narrow-band lasers. The resonances in Figs. 1 and 2 show the inhomogeneous width of the $0 \rightarrow 2$ transition. This result is expected from theory for the present case, where $\Gamma_{12}$ is larger than the inhomogeneous width. For systems with inhomogeneous broadening much larger than $\Gamma_{01}, \Gamma_{02}$, and $\Gamma_{12}$, the ensemble average predicts line-narrowed resonances depending only on the homogeneous parameters if there is positive correlation between $S_{1}$ and $S_{2} \cdot{ }^{1}$ Another interesting feature predicted for spectra in difference-frequency generation are dephasing-induced coherent-emission (DICE) or extra resonances. ${ }^{6}$ These resonances should appear with increasing temperature when pure dephasing pro-

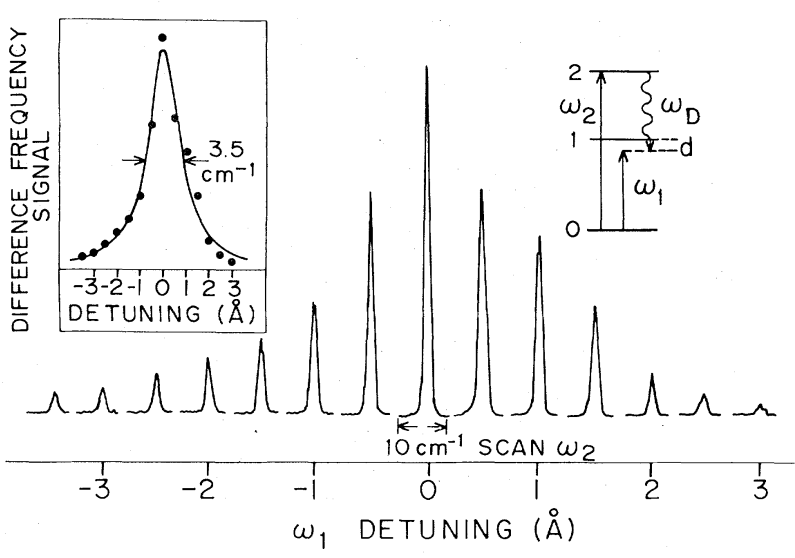

FIG. 2. Resonant difference-frequency generation in dc fields: The $\omega_{2}$ laser scan is shown for various detunings of $\omega_{1}$. The dc field was fixed at $50 \mathrm{kV} / \mathrm{cm}$ directed along the $c^{\prime}$ axis of the naphthalene crystal $\left(\omega_{1}\right.$ and $\omega_{2}$ were polarized along $b$ and $\perp b$, respectively). Inset: The peak intensity of the difference-frequency signal shows a resonance corresponding to the $S_{0} \rightarrow S_{1}$ transition convoluted with the laser widths. The solid line is a fitted Lorenzian. 
cesses set in.

Our measurements have revealed that the dipole moment changes $\Delta \mu_{01}$ and $\Delta \mu_{02}$ are in the same direction, since detuning of $\omega_{1}$ to the lower-energy component of the $0 \rightarrow 1$ transition in the dc field resonantly enhanced the lower-energy component of the sum-frequency signal and vice versa. The absolute values of $\Delta \mu$ for both transitions are known from Stark experiments to be very similar ${ }^{3}$ and hence we expect $\Delta \mu$ to be less than $0.1 \mathrm{D}$.

In conclusion, we report the first observation of high-resolution spectra with use of fully resonant second-order parametric processes. Both sum-and difference-frequency signals have been obtained. The use of centrosymmetric host materials in combination with Stark-effect switching of resonances promises to be very useful and a widely applicable spectroscopic method. The sigmoid shape of the field dependence of the nonlinear signal is unique. Although in this case the levels 0 and 1 were electronic states, the experiment can also be accomplished in the same configuration with vibrational resonances thereby allowing vibrational spectroscopy to be carried out by detecting visible light. The experiments naturally have nanosecond time resolution and involve the detection of coherent signals distant from the laser wavelengths. Extensions to picosecond time resolution are straightforward.

This work was supported in part by the U. S. Army Research Office-Durham through Grant No. DAAG-29-80-C-0014, and in part by the National Science Foundation-Materials Research Laboratory Program through Grant No. DMR8216718. One of us (B.D.) gratefully acknowledges receipt of a research fellowship from the Deutsche Forschungsgemeinschaft.

${ }^{1}$ B. Dick and R. M. Hochstrasser, J. Chem. Phys. 78, 3398 (1983).

${ }^{2}$ R. M. Hochstrasser, Acc. Chem. Res. 6, 263 (1973).

${ }^{3}$ R. M. Hochstrasser and L. J. Noe, J. Chem. Phys. 50, 1684 (1969).

${ }^{4} \mathrm{R}$. W. Hellwarth, in Progress in Quantum Electronics, edited by H. J. Sanders and S. Stenholen (Pergamon, New York, 1977).

${ }^{5}$ R. M. Hochstrasser and T.-Y. Li, J. Mol. Spectrosc. 41, 297 (1972); R. M. Hochstrasser and C. A. Nyi, J. Chem. Phys. 70, 1112 (1979).

${ }^{6}$ Y. Prior, A. R. Bodgan, M. Dagenais, and N. Bloembergen, Phys. Rev. Lett. 46, 111 (1981); J. R. Andrews and R. M. Hochstrasser, Chem. Phys. Lett. 82,381 (1981), and $\underline{83}, 427$ (1981). 